\title{
Epidemiological Trends of Sexually Transmitted Infections with Ureaplasma urealyticum among Women in Cheonan, South Korea: 2006-2017
}

\author{
Jae-Sik Jeon and Jae Kyung Kim* \\ Department of Biomedical Laboratory Science, Dankook University College of Health Sciences, Cheonan 31116, Republic of Korea
}

Received: May 22, 2018 / Revised: August 11, 2018 / Accepted: September 3, 2018

Ureaplasma urealyticum (UU) infection can spread rapidly across populations and is associated with cervical intraepithelial neoplasms, human papillomavirus infections, and newborn mortality. This study aimed to provide information that could be used to protect public health and decrease the incidence and transmission of sexually transmitted infections (STIs), particularly among childbearing women. We examined the epidemiology of UU infection in Cheonan, South Korea. During 2006-2017, 4,050 specimens were submitted for STI screening using a multiplex polymerase chain reaction (PCR) assay. Data were analyzed for UU infection cases using the $R$ statistical program and categorical data were analyzed using the chi-square test, and p-values $<0.05$ were considered statistically significant. Positive PCR results were shown in $17.8 \%$ of the total specimens, in $\mathbf{9 . 0} \%$ of men, and in $\mathbf{1 8 . 7 \%}$ of women. Individuals in their teenaged years and individuals aged 20-29 years accounted for the largest proportions of UU-positive specimens. Although Mycoplasma hominis was the most prevalent bacterium in 2006, it was superseded by UU in 2017. Of the 870 UUpositive specimens, $50.1 \%, 33.1 \%, 13.4 \%$, and $2.8 \%$ had single, double, triple, and quadruple infection, respectively. UU was most common among Korean individuals aged 20-29 years, indicating a high risk of maternal-to-infant transmission that should be addressed through rapid diagnosis, treatment, and management.

Keywords: Sexually transmitted infections, Ureaplasma urealyticum, prevalence, pathogen, infection

\section{Introduction}

Ureaplasma urealyticum (UU) is one of the main pathogens found in women with urogenital infection [1]. In 1977, Taylor-Robinson et al. [2] demonstrated the virulence of UU. By that time, Klein et al. [3] had already reported that it may be a cause of low birth weight in newborns. In addition, several infections resulting from UU have been reported, including salpingitis [4], puerperal sepsis [5], amnionitis [6], tubo-ovaritis [7], and nonspecific prostate urethritis [8]. Quinn et al. [9] reported that it might cause neonatal death or stillbirth.

\section{*Corresponding author}

Tel: +82-41-550-1451, Fax: +82-41-559-7934

E-mail: nerowolf2@dankook.ac.kr

(c) 2018, The Korean Society for Microbiology and Biotechnology
In addition to obstetric complications, UU has recently been shown to have a strong association with cervical intraepithelial neoplasm and human papillomavirus infection. Therefore, rapidly providing treatment to women infected with UU has become more important [10].

UU positivity has been reported to have a higher prevalence than the more commonly known Mycoplasma hominis (MH) [11, 12]. In China, the highest frequency of UU positivity was found in individuals aged 20-29 years and 30-39 years, with both groups accounting for a total of $88.2 \%$ of all UU infections [11]. In addition, the positivity rate was $42.3 \%$ for individuals in their individuals aged 20-34 years [13]. In Korea, there are reports that the rate of $\mathrm{UU}$ infection is higher than that of $\mathrm{MH}$ [14, 15]; $47.4 \%$ and $37.9 \%$ positivity rates have been 
reported for individuals in their teens and individuals aged 20-29 years, respectively [16].

These sexually transmitted infections (STIs), which occur during reproductive ages, can spread quickly in many populations and can have a fatal effect on newborns. Therefore, this study aimed to investigate the trend of UU infection in South Korea. The ultimate goal was to provide information that could be used to protect public health and decrease the incidence and transmission of STIs, particularly among childbearing women. We want to provide basic data that can be used for further research.

\section{Materials and Methods}

From August 2006 to November 2017, 4,050 specimens were collected at Dankook University Hospital in Cheonan province, Korea. Specimens were collected from female patients who were 10 years of age or older who visited the hospital for STI testing in obstetrics and gynecology and emergency room.

The samples were analyzed for the presence of several pathogens using a multiplex polymerase chain reaction (PCR) assay. PCR amplification was performed using the Seeplex STD6 Detection Kit (Seegene, Korea), according to the manufacturer's instructions, using a PTC 200 PCR system (MJ Research, USA). The PCR kit tested for six species: Chlamydia trachomatis (CT), Mycoplasma genitalium (MG), MH, Neisseria gonorrhoeae (NG), Trichomonas vaginalis (TV), and UU.

In accordance with the manufacturer's instructions, a QIAamp DNA Mini Kit (Qiagen, Düren, Germany) was used to prepare genomic DNA for the multiplex PCR assay. Approximately $200 \mu \mathrm{l}$ of each sample was used as starting material for the nucleic acid isolation. Concentrations of the extracted DNA samples (undiluted) were measured using a spectrophotometer (NanoDrop 1000; Thermo Fisher Scientific, USA). The undiluted samples contained $>20 \mu \mathrm{g} / \mathrm{ml}$ of DNA.

The initial PCR step was performed at $94^{\circ} \mathrm{C}$ for $15 \mathrm{~min}$ then underwent 40 cycles under the following conditions: $94^{\circ} \mathrm{C}$ for $30 \mathrm{~s}, 63^{\circ} \mathrm{C}$ for $90 \mathrm{~s}$, and $72^{\circ} \mathrm{C}$ for $90 \mathrm{~s}$. The final cycle was followed by an extension step at $72{ }^{\circ} \mathrm{C}$ for $10 \mathrm{~min}$ to complete any partial polymerizations. Positive and negative PCR controls containing standardized viral RNA extracts and nuclease-free water were included in each run. The kit includes amplification of the Arabidopsis cellulose synthase (CesA3) gene as an internal control (IC), which is designed to detect the presence of PCR inhibitors. An IC was included in the PCR mix to detect the presence of PCR inhibitors. PCR products were separated using QIAxcel Advanced (Qiagen) with QIAxcel DNA Kits (Qiagen).

We downloaded the results from the automated computer system in the hospital. Data were analyzed for the presence of STI using the R statistical program (version 3.3.3, Comprehensive R Archive Network; https://www.rproject.org) and presented as the median and range. The chi-square test was used to analyze categorical data.

The study protocol was approved by the institutional review board (IRB) of Dankook University, as retrospectively registered (IRB Approval No: 2017-10-004). The study was conducted in conformance with the tenets of the Declaration of Helsinki.

\section{Results}

The average age of patients who submitted specimens was 36.3 years. The proportion of samples that tested positive for STIs is $38.7 \%(1,569 / 4,050)$. Of the total samples, $19.2 \%(779 / 4,050)$ tested positive for $\mathrm{MH}, 19.1 \%$ (775/4,050) tested positive for UU, and 10.5\% (427/4,050) tested positive for CT (Table 1). Of the 775 samples that tested positive for UU, 49.2\% (381) were positive for UU alone and 50.8\% (394/775) had a co-infection (33.4\% with double infection and $17.4 \%$ with triple or more infection) with another pathogen (Table 1).

Individuals aged 20-29 years accounted for the greatest number of UU-positive specimens (214/775), followed by individuals aged 10-19 years (174/775) and individuals aged 40-49 years (152/775). Of the age groups, the highest rate of UU positivity was observed for individuals aged 10-19 years (35.9\%, 174/485), followed by individuals aged $20-29$ years $(22.3 \%, 214 / 960)$, and individuals aged $50-59$ years $(16.5 \%, 63 / 382)$ (Fig. 1$)$.

Regarding trends from 2007 to 2016 , UU was most commonly detected in 2014 (129/775, 16.6\%), and the highest positivity rate was $25.3 \%$ (129/510) in 2014 (Table 1).

The highest number of positive specimens was in the third quarter of 2014. The average age was the highest in the fourth quarter of 2010 and the lowest in the first 
Table 1. Detection number, positive rate and average age of STI pathogens in the submitted specimens.

\begin{tabular}{|c|c|c|}
\hline & $\begin{array}{l}\text { Number of specimens } \\
\text { (positive rate) }\end{array}$ & Average age \\
\hline Total & $4,050(100.0 \%)$ & 36.3 \\
\hline Positive & 1,569 (38.7\%) & 33.3 \\
\hline CT & $427(10.5 \%)$ & 27.1 \\
\hline $\mathrm{MH}$ & 779 (19.2\%) & 35.0 \\
\hline UU & 775 (19.1\%) & 32.4 \\
\hline Other pathogens ${ }^{a}$ & 294 (7.3\%) & 31.4 \\
\hline \multicolumn{3}{|l|}{ Infection type of UU } \\
\hline Single infection & $381(49.2 \%)$ & 33.7 \\
\hline Double infection & 259 (33.4\%) & 32.9 \\
\hline Triple or more infection & 135 (17.4\%) & 27.8 \\
\hline Year & $\begin{array}{l}\text { Number of positive / } \\
\text { submitted } \\
\text { specimens } \\
\text { (positive rate) }\end{array}$ & $\begin{array}{l}\text { Average age of } \\
\text { UU positive } \\
\text { specimens }\end{array}$ \\
\hline 2006 & 15 / $73(20.5 \%)$ & 38.3 \\
\hline 2007 & 47 / 259 (18.1\%) & 39.4 \\
\hline 2008 & 27 / 216 (12.5\%) & 40.1 \\
\hline 2009 & 25 / 223 (11.2\%) & 36.4 \\
\hline 2010 & 13 / 182 (7.1\%) & 38.2 \\
\hline 2011 & 65 / 344 (18.9\%) & 34.5 \\
\hline 2012 & 73 / 371 (19.7\%) & 28.4 \\
\hline 2013 & 106 / 469 (22.6\%) & 31.9 \\
\hline 2014 & 129 / 510 (25.3\%) & 31.5 \\
\hline 2015 & 118 / 528 (22.3\%) & 33.0 \\
\hline 2016 & 86 / 531 (16.2\%) & 31.2 \\
\hline 2017 & 71 / 344 (20.6\%) & 30.9 \\
\hline
\end{tabular}

aOther pathogens: MG, Mycoplasma genitalium; NG, Neisseria gonorrhoeae; TV, Trichomonas vaginalis. Abbreviations: CT, Chlamydia trachomatis; MH, Mycoplasma hominis; UU, Ureaplasma urealyticum.

quarter of 2012. The number of positive specimens and positive rates tended to increase $(p<0.000, p=0.005)$, but average ages tended to decrease $(p=0.007)$ every year (Fig. 2).

\section{Discussion}

UU has been believed to be pathogenic since its discovery, but there has been controversy over whether it is virulent, as it is known to colonize the urogenital tracts of more than $20 \%$ of women [17]. Recently, genital mycoplasma infection has become known as an STIs, and is

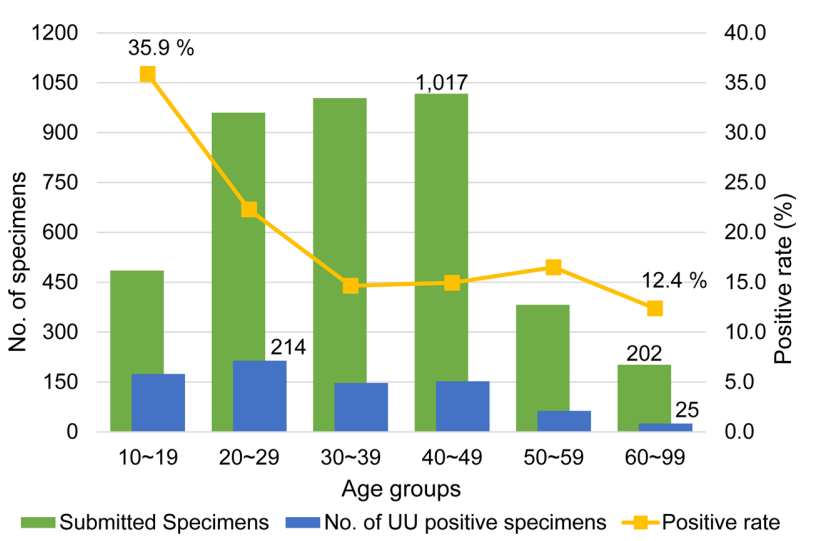

Fig. 1. Distribution of specimens according to UU detection by age group. Abbreviation: UU, Ureaplasma urealyticum.

considered to be of similar importance to previously recognized STIs, such as gonorrhea and syphilis or CT infection [18]. Urogenital tract infections are a major cause of morbidity in sexually active individuals worldwide; therefore, the World Health Organization has ranked STIs second in importance after cancer in an analysis of treatable diseases in women [19].

Of the 4,050 samples that were tested during the approximately 11-year duration of this study, $38.7 \%$ $(1,569 / 4,050)$ were positive for STIs. Of these, UU accounted for the second largest number of positive specimens $(775 / 4,050,19.1 \%)$. This percentage is similar to corresponding values previously reported by Lee et al. (21.2\%) [20] and Moon et al. (17.6\%) [21]. Of the 775 samples that tested positive for UU, 49.2\% (381/775) were single infections, $33.4 \%$ (259/775) were double infections, and $17.4 \%(135 / 775)$ were triple or more infections. Samra et al. [12] reported a similar rate of single infections in their study at $45.1 \%$. Although recent increases in the incidence of UU infection have been reported, there are many regional differences; thus, a global analysis is needed.

In a previous study, Zhu et al. [13] reported that the UU positivity rate was highest in the 30-39 age group at $50.9 \%$ (30-34 years old, 35.8\%; 35-40 years, $15.1 \%$ ). In 2014, Wang et al. [11] also reported that the highest UU positivity rate was among those aged 30-39 years at $50.2 \%$. Meanwhile, in 2010, Bayraktar et al. [22] reported the highest UU positivity rate among those aged 18-29 years at $55.5 \%$ (for 18-24 years, the corresponding value was $29.6 \%$; for $25-29$ the corresponding value was 


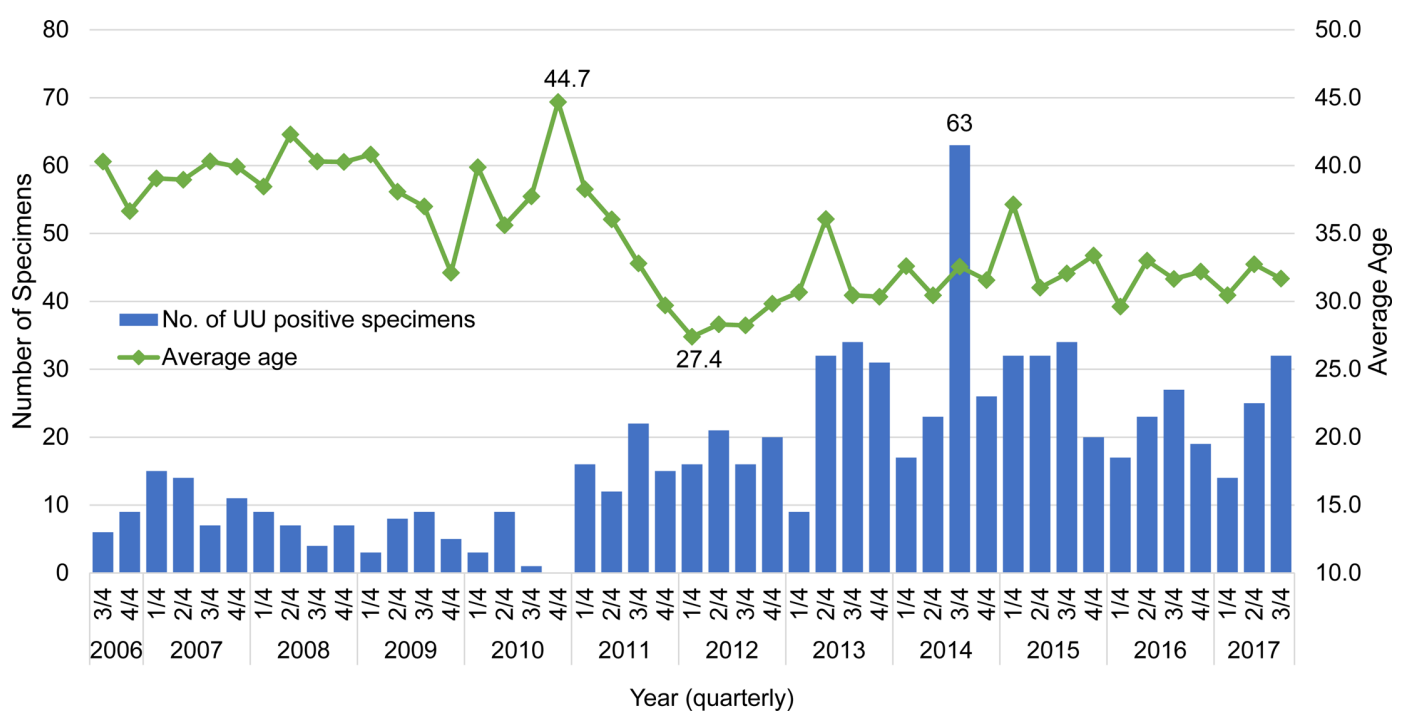

Fig. 2. Change of UU positive specimen number and average age by annual quarterly. Abbreviation: UU, Ureaplasma urealyticum.

$25.29 \%$ ). In this study, the highest positivity rate of UU was in the teenager group $(35.9 \%, 174 / 485)$, and the highest number of positive specimens was in the 20-29 age group $(27.6 \%, 214 / 775)$. While differences with respect to the period and region investigated in these studies must be acknowledged, the findings all indicate that the average age of patients with UU infection appears to be decreasing.

According to the Korea Centers for Disease Control [23], the total number of reported STIs cases decreased from 24,583 in 2002 to 9,387 in 2006. Thereafter, the number of cases continued to decrease, falling from 8,983 in 2007 to 7,422 in 2010 . However, this decreasing trend ended in 2011. Since then, the incidence of UU infection increased until 2016. These changes are similar to the results observed in our study. Analysis of annual data showed that the highest number of UU positive specimens and the highest positivity rate occurred in the third quarter of $2014(8.1 \%, 63 / 775)$. The UU positivity rate increased from $20.5 \%$ in 2006 to $20.6 \%$ in 2014. Although there appears to be no change, the positive rate has increased by $0.18 \%$ per quarter per year. In addition, Wang et al. [11] also reported an increase from $42.2 \%$ in 2005 to $49.3 \%$ in 2007 and $47.4 \%$ in 2008 . Although MH was initially perceived to be more common, the proportion of UU-positive cases has consistently increased in the last five years. The cause of this phenomenon needs further investigation.
There are some limitations to this study. First, it was conducted in only one region, and further research is needed in various regions. Second, the population within the region was not uniformly examined, and a largersize multicenter study is needed. Finally, no further experiments were performed because this was a retrospective study.

In conclusion, our results show that UU infection comprises a large proportion of STIs cases in Korea. The number of UU infections was highest for individuals in the 20-29-year age group and the positive rate was highest in the 10-19-year age group. Given that they are at a childbearing age, the risk of maternal-to-infant infection transmission is high among younger people compared to the elderly. Therefore, rapid diagnosis, treatment, and management of UU infection, particularly in young individuals, is imperative to curb the high rate of infection.

\section{Conflict of Interest}

The authors have no financial conflicts of interest to declare.

\section{References}

1. Zhang Y, Hua C, Li SL. 2018. The relationship between the biovars and the antimicrobial resistance of Ureaplasma urealyticum in female patients with urogenital infections. J. Clin. Lab. Anal. 32: e22211.

2. Taylor-Robinson D, Csonka GW, Prentice MJ. 1977. Human intra- 
urethral inoculation of Ureaplasmas. Q. J. Med. 46: 309-326.

3. Klein JO, Buckland D, Finland M. 1969. Colonization of newborn infants by mycoplasmas. N. Engl. J. Med. 280: 1025-1030.

4. Mårdh PA, Weström L. 1970. T-mycoplasmas in the genito-urinary tract of the female. Acta. Pathol. Microbiol. Scand. B. Microbiol. Immunol. 78: 269.

5. Sompolinsky D, Solomon F, Leiba H, Caspi E, Lewinsohn G, Almog C. 1971. Puerperal sepsis due to T-strain Mycoplasma. Isr. J. Med. Sci. 7: 745-748.

6. Caspi E, Herczeg E, Solomon F, Sompolinsky D. 1971. Amnionitis and T Strain mycoplasmemia. Am. J. Obstet. Gynecol. 111: 11021106.

7. Braun P, Besdine R. 1973. Tuboovarian abscess with recovery of Tmycoplasma. Am. J. Obstet. Gynecol. 117: 861-862.

8. Weidner W, Brunner H, Krause W, Rothague CF. 1978. The importance of Ureaplasma urealyticum in non-specific prostato-urethritis. Dtsch. Med. Wochenschr. 103: 465-470.

9. Quinn PA, Butany J, Chipman M, Taylor J, Hannah W. 1985. A prospective study of microbial infection in stillbirths and early neonatal death. Am. J. Obstet. Gynecol. 151: 238-249.

10. Biernat-Sudolska M, Szostek S, Rojek-Zakrzewska D, Klimek M, Kosz-Vnenchak M. 2011. Concomitant infections with human papillomavirus and various mycoplasma and Ureaplasma species in women with abnormal cervical cytology. Adv. Med. Sci. 56: 299-303.

11. Wang QY, Li RH, Zheng LQ, Shang XH. 2016. Prevalence and antimicrobial susceptibility of Ureaplasma urealyticum and Mycoplasma hominis in female outpatients, 2009-2013. J. Microbiol. Immunol. Infect. 49: 359-362.

12. Samra Z, Rosenberg S, Madar-Shapiro L. 2011. Direct simultaneous detection of 6 sexually transmitted pathogens from clinical specimens by multiplex polymerase chain reaction and autocapillary electrophoresis. Diagn. Microbiol. Infect. Dis. 70: 17-21.

13. Zhu X, Li M, Cao H, Yang X, Zhang C. 2016. Epidemiology of Ureaplasma urealyticum and Mycoplasma hominis in the semen of male outpatients with reproductive disorders. Exp. Ther. Med.
12: $1165-1170$.

14. Chung SH, Kim TH, Yeo SJ, Kim JS, Kim HM, Jung EJ, et al. 2007. Isolation of Mycoplasma hominis and Ureaplasma urealyticum from the uterine cervix and vaginal wall of pregnant women and correlation with perinatal outcome. Korean J. Fetal. Med. 2: 1-6.

15. Lee MY, Kim MH, Lee WI, Kang SY, Jeon YL. 2016. Prevalence and antibiotic susceptibility of Mycoplasma hominis and Ureaplasma urealyticum in pregnant women. Yonsei Med. J. 57: 1271-1275.

16. Kim JK. 2013. Epidemiological trends of sexually transmitted infections among women in Cheonan, South Korea, 2006-2012. J. Microbiol. Biotechnol. 23: 1484-1490.

17. Taylor-Robinson D, Furr PM, Liberman MM. 1984. The occurrence of genital mycoplasmas in babies with and without respiratory distress. Acta. Paediatr. Scand. 73: 383-386.

18. Chung SH, Kim TH, Yeo SJ, Kim JS, Kim HM, Jung EJ et al. 2007. Isolation of Mycoplasma hominis and Ureaplasma urealyticum from the Uterine Cervix and Vaginal Wall of Pregnant Women and Correlation with Perinatal Outcome. Korean J. Fetal Med. 2: 16.

19. Yamazaki T, Matsumoto M, Matsuo J, Abe K, Minami K, Yamaguchi H. 2012. Frequency of Chlamydia trachomatis in Ureaplasmapositive healthy women attending their first prenatal visit in a community hospital in Sapporo, Japan. BMC. Infect. Dis. 12: 82.

20. Lee SJ, Park DC, Lee DS, Choe HS, Cho YH. 2012. Evaluation of Seeplex ${ }^{\circledast}$ STD6 ACE Detection kit for the diagnosis of six bacterial sexually transmitted infections. J. Infect. Chemother. 18: 494-500.

21. Moon SJ, Choi J-E, Park K-I. 2013. Comparison of the Anyplex II STI-7 and Seeplex STD6 ACE Detection kits for the detection of sexually transmitted infections. J. Lab. Med. Qual. Assur. 35: 87-92.

22. Bayraktar MR, Ozerol IH, Gucluer N, Celik O. 2010. Prevalence and antibiotic susceptibility of Mycoplasma hominis and Ureaplasma urealyticum in pregnant women. Int. J. Infect. Dis. 14: e90-e95.

23. Korea Centers for Disease Control and Prevention. Infectious disease portal. Korea Centers for Disease Control and Prevention. Available from http://www.cdc.go.kr/npt/biz/npp/iss/stisStatisticsMain.do Accessed Jan. 7, 2018. 